Publisher homepage: www.universepg.com, ISSN: 2707-4625 (Online) \& 2707-4617 (Print) https://doi.org/10.34104/ijmms.020.087092

International Journal of Material and Mathematical Sciences

Journal homepage: www.universepg.com/journal/ijmms

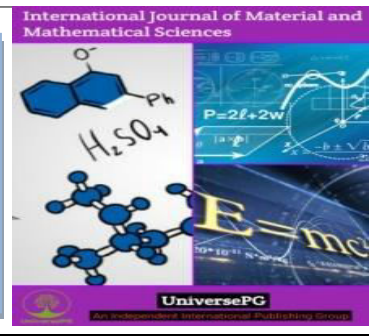

\title{
The Metric of Space-Time Curvature in a Weak Gravitational Field and it's Consequence in Newtonian Approximation
}

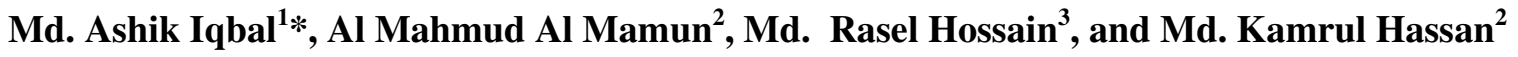 \\ ${ }^{1}$ Dept. of Applied Mathematics, University of Rajshahi, Rajshahi, Bangladesh, ${ }^{2}$ Dept. of Computer Science and Engineering, \\ Islamic University, Bangladesh; and ${ }^{3}$ Dept. of Statistics, Noakhali Science and Technology University, Noakhali, \\ Bangladesh. \\ *Correspondence: ashikiqbalmath@gmail.com (Md. Ashik Iqbal, Dept. of Applied Mathematics, University of Rajshahi, \\ Rajshahi, Bangladesh).
}

\begin{abstract}
In Newtonian mechanics, space and time are separate but in General Relativity is unified. It is considered that the space in the weak-field approximation is quasi-static and it arises from a perfect field whose particles have very small velocity in comparison to light velocity in this coordinate system and the metric is a gravitational potential tensor of rank two which implies the field of empty space. If each point of an area in N-dimensional space there existed a corresponding definite tensor, where the components of the tensor are the function of space and space acts as the strong or weak gravitational field.
\end{abstract}

Keywords: Minkowskian metric, Gravitational field, Space-time metric, Geodesic equation, and Manifolds.

\section{INTRODUCTION:}

General Relativity is the relativistic theory of strong gravitational space whereas the special theory of relativity only accounts for the weak field of the region which is called free space, where gravitational effects can be neglected (Veltman, 1975; Richard, 1983; Resnick, 1968; Hughton, 1990). In those systems the law of inertia holds good and the physical laws retain the same form (Alam and Alam, 2020). In some freely - declining position and make a large structure out of the solid bar, at a certain interval off, the freelydeclining body will have resembled that they are accelerating with regard to this frame of reference and according to Einstein's equivalence principle, the physical laws of nature convert to those of Newtonian Relativity in an infinitesimal region of space-time which is known to non-relativistic limit (Narlikar, 1978; Boss, 1980; Relativity, 1964; Stephani, 2004).

UniversePG I www.universepg.com
These considerations lead to the vital concept of gravity force which is not actually a force but a manifestation of space-time curvature. The crucial difference between strong and weak gravitational fields is that in a field space the result of parallel transporting a vector from one point to another will depend on the path taken between the points (Rinder, 2001; Ohanian and Ruffini, 1994).

In the transformation of vectors in a weak field, initial and final vectors remain the same at a time. But in a strong field initial and final vectors are different. The change of the vector $A_{\mu}$ is $d A_{\mu}$ which can be divided into two components. One component $\partial A_{\mu}$ due to the change of direction of the axis of coordinates and another is $\partial^{\prime} A_{\mu}$ due to the change of the intrinsic value of the vector (Caroll, 1997; Boss, 1980). 
i.e. $d A_{\mu}=\partial A_{\mu}+\partial^{\prime} A_{\mu}$

In weak gravitational field there is no changed of vector with the change of axis. Hence, $\partial A_{\mu}=0$. Which implies that the direction of vector is constant in weak field and the metric is the function of gravitational potential (Will, 2018).

\section{Coordinate System}

To explain the motion of a particle in Newtonian mechanics, a few numbers of coordinate systems are used. To take the distance between two adjacent points is the best medium to identify the coordinate system and this interval between two adjacent points is called a metric or line element. In three dimensional space, the interval of the points-

$$
\left(x^{1}, x^{2}, x^{3}\right), \text { and }\left(x^{1}+d x^{1}, x^{2}+d x^{2}, x^{3}+d x^{3}\right)
$$

In Cartesian coordinates, system is given by (Stephani, 2004; Goyal and Gupta, 1942)

$$
d s^{2}=\left(d x^{1}\right)^{2}+\left(d x^{2}\right)^{2}+\left(d x^{3}\right)^{2} .
$$

In cylindrical coordinates $(\rho, \varphi, z)$ it is

$$
d s^{2}=d \rho^{2}+\rho^{2} d \varphi^{2}+d z^{2} .
$$

And in spherical polar coordinates $(r, \theta, \varphi)$ it is

$$
d s^{2}=(d r)^{2}+(r d \theta)^{2}+(r \sin \theta d \varphi)^{2}
$$

So, in any coordinate system the square of the space between two points be -

$$
d s^{2}=g_{\mu \nu}(\mathrm{x}) d x^{\mu} d x^{\nu}
$$

$[\mu, v=1,2,3]$

Now, In Cartesian coordinates:

$$
g_{11}=g_{22}=g_{33}=1 \text {. }
$$

In cylindrical coordinates:

$$
g_{11}=g_{33}=1, g_{22}=\rho \text {. }
$$

In spherical polar coordinates:

$$
g_{11}=1, g_{22}=r, g_{33}=r \sin \theta
$$

And the others are $g_{i j}=0[$ for $i \neq j]$.

Here the form (4) is called fundamental metric form.

\section{Coordinate Transformations}

Suppose in two dimensional coordinates system the Cartesian and polar coordinate of a point are $(x, y)$ and $(r, \theta)$, then written the coordinate transformations are below (Ansari, 2020).

$$
\begin{aligned}
& x=r \cos \theta=f(r, \theta), \\
& y=r \sin \theta=f(r, \theta) .
\end{aligned}
$$

Inversely,

$$
\begin{aligned}
& r=\sqrt{x^{2}+y^{2}}=F(x, y) \\
& \theta=\tan ^{-1}\left(\frac{y}{x}\right)=F(x, y) .
\end{aligned}
$$

From the above relations we see that a coordinate of one reference frame is the function of all coordinates of another frame of reference. Similarly, we can show the same transformation in 3-D system. In the basis of this concept into the $N$-dimensional system, if the coordinates of a point in two difference reference frame are -

$\left(x^{1}, x^{2}, x^{3}, \ldots, x^{N}\right)$ and,

$\left(\bar{x}^{1}, \bar{x}^{2} \bar{x}^{3}, \ldots, \bar{x}^{N}\right)$,

Then we have $N$-independent relations (Caroll, 1997).

$\bar{x}^{i}=\bar{x}^{i}\left(x^{1}, x^{2}, x^{3}, \ldots, x^{N}\right)$,

Where, $i=1,2,3, \ldots, N$.

Inversely,

$x^{i}=x^{i}\left(\bar{x}^{1}, \bar{x}^{2}, \bar{x}^{3}, \ldots, \bar{x}^{N}\right)$.

Differentiating (9) partially we get

$d \bar{x}^{i}=\frac{\partial \bar{x}^{i}}{\partial x^{1}} d x^{1}+\frac{\partial \bar{x}^{i}}{\partial x^{2}} d x^{2}+\cdots+\frac{\partial \bar{x}^{i}}{\partial x^{N}} d x^{N}$,

Or,

$d \bar{x}^{i}=\sum_{j=1}^{N} \frac{\partial \bar{x}^{i}}{\partial x^{j}} d x^{j}$.

By using Einstein's summation convention we have,

$d \bar{x}^{i}=\frac{\partial \bar{x}^{i}}{\partial x^{j}} d x^{j}$.

Replacing the vector $d \bar{x}^{i}$ by $\bar{A}^{\mu}$ and $d x^{j}$ by $A^{v}$ we have,

$\bar{A}^{\mu}=\frac{\partial \bar{x}^{\mu}}{\partial x^{v}} A^{v}$. 
Inversely,

$$
A^{v}=\frac{\partial x^{v}}{\partial \bar{x}^{\mu}} \bar{A}^{\mu} .
$$

Relations (14) and (15) are defined to the transformation of coordinates from one frame of reference to another.

\section{Manifolds:}

Manifold refers to the various types of surfaces such as the plane is two dimensional manifolds. A manifold is one of the most fundamental concepts in mathematics and physics. Consider the set $\mathrm{U}(\mathrm{P})$ which is the neighborhood of $\mathrm{P}$ and $\mathrm{P}$ is any point on the Manifold $\mathrm{M}$ which can be explained by the $\mathrm{N}$ number of elements $\left(\mathrm{x}^{1}, \mathrm{x}^{2}, \mathrm{x}^{3}, \ldots \ldots \ldots \mathrm{x}^{\mathrm{N}}\right)$, Now, in Euclidean space the distance between two points $\mathrm{P}_{\mathrm{A}}$ and $\mathrm{P}_{\mathrm{B}}$ be (Foster and Nightingale, 1994; Caroll, 1997).

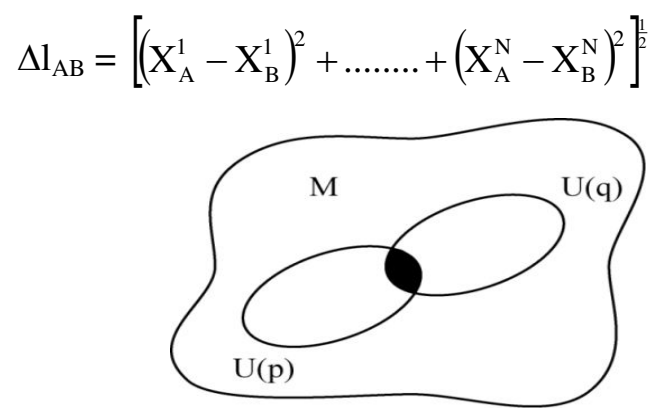

Fig 1: Two dimensional manifolds.

Another point $\mathrm{q}$ and its neighborhood, open set $\mathrm{U}(\mathrm{q})$ can be explained by the $\mathrm{N}$-number of real quantities $\left(y^{1}, y^{2}, \ldots . y^{N}\right)$. Now the points of the common region of these two neighborhoods can be expressed through any of them coordinate systems. In this case, the coordinate transformation is -

$$
y^{i}=y^{i}\left(x^{1}, x^{2}, x^{3}, \ldots x^{N}\right)[i=1,2,3, \ldots N]
$$

The transformation from one coordinates system to another coordinates system is called one-one mapping or direct transformation. So, we can define the manifold as a connected Housdorf space which is locally Euclidean.

\section{Space-time Metric:}

The special relativistic line element (or metric), when Cartesian coordinates $(c t, x, y, z)$ are used, is given by

$$
d s^{2}=\eta_{\mu \nu} d x^{\mu} d x^{v}=c^{2} d t^{2}-d x^{2}-d y^{2}-d z^{2} .
$$

Where, $\eta_{\mu v}$ is the flat-space Minkowskian metric tensor. If the inertial coordinate frames will be changed into another then in Lorentz transformation, the spacetime metric (18) does not change (Stephani, 2004; Ellis and Williams, 1988).

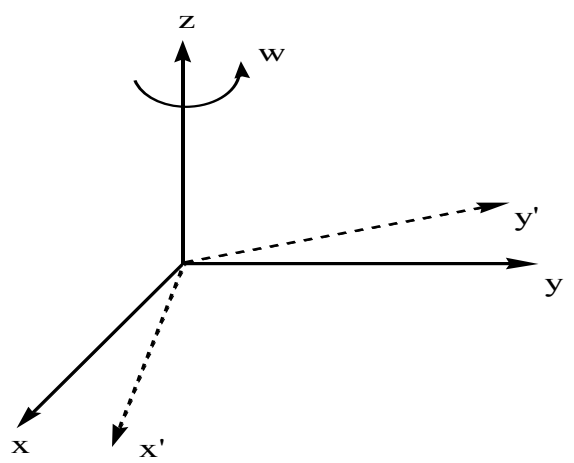

Fig 2: Inertial frame into a non-inertial frame and the revolution.

Consider the changes from an inertial frame into a noninertial frame and the revolution is taken concerning the z-axis (Synge and Schild, 1949). Now, the conversion equations are -

$$
\left.\begin{array}{c}
x=x^{\prime} \cos \omega t-y^{\prime} \sin \omega t \\
y=x^{\prime} \sin \omega t+y^{\prime} \cos \omega t
\end{array}\right\}
$$

Where, $\omega$ is the angular velocity of rotation. From (19) we have,

$d x=d x^{\prime} \cos \omega t-x^{\prime} \omega \sin \omega t d t-\sin \omega t d y^{\prime}-y^{\prime} \omega \cos \omega t d t$

$d y=\sin \omega t d x^{\prime}+x^{\prime} \omega \cos \omega t d t+\cos \omega t d y^{\prime}-y^{\prime} \omega \sin \omega t d t$

$d z=d z^{\prime}$

Putting these values in (18) we have,

$$
\begin{gathered}
d s^{2}=\left[c^{2}-\omega^{2}\left(x^{\prime 2}+y^{\prime 2}\right)\right] d t^{2} \\
+2 \omega d t\left(y^{\prime} d x^{\prime}-x^{\prime} d y^{\prime}\right) \\
-\left(d x^{\prime}+d y^{\prime}+d z^{\prime}\right)^{2} \\
=g_{\mu \nu} d x^{\mu} d x^{\nu .}
\end{gathered}
$$

Where the components are,

$$
\begin{array}{ll}
g_{00}=\frac{\left[c^{2}-\omega^{2}\left(x^{\prime 2}+y^{\prime 2}\right)\right]}{c^{2}}, & g_{11}=g_{22}=g_{33}-1, \\
g_{01}=g_{10}=\omega y^{\prime}, & g_{02}=g_{20}=-\omega x .
\end{array}
$$

And all other components of $g_{\mu v}$ vanish and this is different from (18). In the general case, when noneinertial coordinate frames are used, the line element will have following expression (Anderson, 1975). 
$d s^{2}=g_{\mu \nu} d x^{\mu} d x^{v}$.

Where, $g_{\mu \nu}(x)$ are ten functions of,

$$
\mathrm{X} \equiv\left(x^{0}, x^{1}, x^{2}, x^{3}\right)
$$

With $g_{\mu \nu}=g_{v \mu}$.

In Cartesian coordinates

$$
x^{0}=c t, x^{1}=x, x^{2}=y, x^{3}=z
$$

Now, we can use non-Cartesian coordinates and in that case, the coordinates $x^{1}, x^{2}$ and $x^{3}$ describe curvilinear coordinates (Weinberg, 1972). The metric tensor $\mathrm{g}_{\mu \nu}$ describes the none-inertial field of forces are equivalent to gravitational fields. So, the space-time metric in general relativity has the more general form given by (24), (Max, 1964). Here, $g_{\mu \nu}(x)$ represents the gravitational potential (field). Using tensor transformation we can show that,

$$
\bar{g}_{\mu \nu}(\bar{x}) d \bar{x}^{\mu} d \bar{x}^{\nu}=g_{\mu \nu} d x^{\mu} d x^{\nu}=d s^{2}
$$

\section{Parallel Transformation of Vector and Covariant Derivative}

The concept of moving a vector along a path keeping constant all the while is known as parallel transport. To subtract of two vectors on a curve space they must be taken at the same position. Now, to bring one vector at the position of another vector there must be needed to the parallel transformation of that vector. Suppose a vector $A_{\mu}$ is taken from the position $x^{\mu}$ to the nearest position $x^{\mu}+d x^{\mu}$ and at this position the vector is $A_{\mu}+d A_{\mu}$.

In Cartesian coordinates system the change of vector $d A_{\mu}$ is zero, but in general curvilinear coordinate it is not zero (Stephani, 2004; Caroll, 1997).

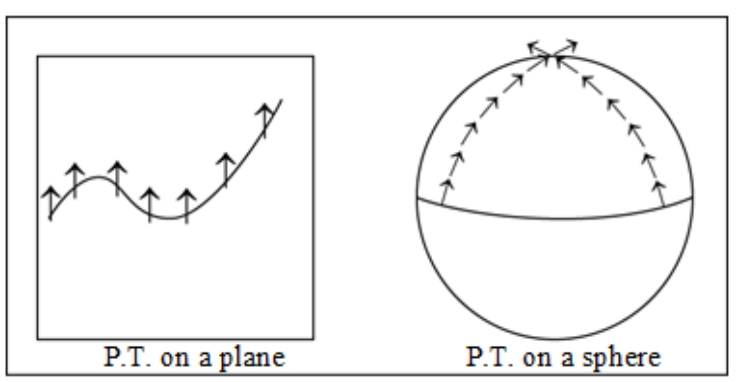

Fig 3: Parallel Transformation of Vector and Covariant Derivative.

\section{Newtonian Approximation}

Consider the motion of a test particle in case of a weak static field. The motion of a test particle is governed by geodesic equations as given below,

$$
\frac{d^{2} x^{\alpha}}{d s^{2}}+\Gamma_{\mu \nu}^{\alpha} \frac{d x^{\mu}}{d s} \frac{d x^{v}}{d s}=0
$$

Since the field is static, i.e. it does not Change with time. Hence velocity components can be taken as (Weinberg, 1972; Caroll, 1997).

$$
\frac{d x^{1}}{d s}, \frac{d x^{2}}{d s}, \frac{d x^{3}}{d s}=0 ; \text { and } \frac{d x^{0}}{d s}=1
$$

Our co-ordinates are Galilean co-ordinates

$$
x^{0}=c t, x^{1}=x, x^{2}=y, x^{3}=z
$$

Now we use the metric $g_{\mu \nu}$ for the curve space and the metric $\eta_{\mu v}$ for the flat space, then a weak static field as Characterized by taking

$$
g_{\mu \nu}=\eta_{\mu \nu}+\lambda_{1} g_{\mu \nu}+\lambda_{2}^{2} g_{\mu \nu}+\ldots
$$

Where the parameters $\lambda$ 's are taken to be small. Neglecting the $2^{\text {nd }}$ and higher order term of $\lambda$ we have

$$
g_{\mu \nu} \approx \eta_{\mu \nu}+\lambda_{1} g_{\mu \nu}=\eta_{\mu \nu}+h_{\mu \nu} \text {. }
$$

Where the quantities $h_{\mu \nu}=\lambda_{1} g_{\mu \nu}$ are small but not so small that they may be neglected and

$$
\eta_{\mu \nu}=\left(\begin{array}{cccc}
1 & 0 & 0 & 0 \\
0 & -1 & 0 & 0 \\
0 & 0 & -1 & 0 \\
0 & 0 & 0 & -1
\end{array}\right)
$$

We have,

$$
\begin{aligned}
& \eta_{00}=1, \eta_{11}=\eta_{22}=\eta_{33}=-1 \text { and, } \\
& \eta_{\mu \nu}=0=g_{\mu \nu}[\text { For } \mu \neq v] .
\end{aligned}
$$

Again,

$$
\begin{aligned}
& \mathrm{ds}^{2}=g_{\mu \nu} \mathrm{dx}^{\mu} \mathrm{dx}^{v} \text { gives } \\
& 1=g_{\mu \nu} \frac{\mathrm{dx}^{\mu}}{\mathrm{ds}} \frac{\mathrm{dx}^{v}}{\mathrm{ds}} \\
& \Rightarrow 1=g_{00} \frac{d x^{0}}{d s} \frac{d x^{0}}{d s}\left[\because \frac{d x^{1}}{d s}=\frac{d x^{2}}{d s}=\frac{d x^{3}}{d s}=0\right] \\
& =\left(\eta_{00}+h_{00}\right) \frac{d t}{d s} \cdot c \frac{d t}{d s} \\
& =\left(1+h_{00}\right) c^{2}\left(\frac{d t}{d s}\right)^{2} \\
& {\left[\because \eta_{00}=1, x^{0}=c t\right]}
\end{aligned}
$$


$\Rightarrow c^{2}\left(\frac{d t}{d s}\right)^{2}=\left(1+h_{00}\right)^{-1}=\left(1-h_{00}+h_{00}^{2} \ldots\right)$

Neglecting the $2^{\text {nd }}$ and higher order term of $h_{00}$

$$
\left(\frac{d t}{d s}\right)^{2} \approx \frac{1}{c^{2}}-\frac{h_{00}}{c^{2}}
$$

Neglecting $\frac{h_{00}}{c^{2}}$ as a very small quantity we have (Ellis and Williams, 1988; Goyal and Gupta, 1942).

$$
\frac{\mathrm{dx}^{0}}{\mathrm{ds}}=1
$$

From equation (26) we have (Synge and Schild, 1949)

$$
\begin{aligned}
& \frac{d^{2} x^{\alpha}}{d s^{2}}+\Gamma_{00}^{\alpha} \frac{d x^{0}}{d s} \frac{d x^{0}}{d s}=0 \\
\Rightarrow & \frac{d^{2} x^{\alpha}}{d s^{2}}=-\Gamma_{00}^{\alpha}\left(\frac{d x}{d s}\right)^{2}=-\Gamma_{00}^{\alpha} \\
\Rightarrow & \frac{d^{2} x^{\alpha}}{d t^{2}}=-c^{2} \Gamma_{00}^{\alpha} .
\end{aligned}
$$

Now, for $\alpha=0$

$$
\Gamma_{00}^{0}=0 .
$$

Therefore,

$$
\frac{d^{2} x^{\alpha}}{d t^{2}}=-c^{2} \Gamma_{00}^{\alpha}[\text { for } \alpha=1,2,3] .
$$

We have

$$
\Gamma_{\mu \nu}^{\alpha} g^{\mu \beta}[\mu \nu, \beta]=g^{\alpha \alpha} \Gamma_{\mu v, \alpha}
$$

[By summation convention]

$$
\begin{aligned}
& \Gamma_{00}^{\alpha}=\frac{1}{2} g^{\alpha \alpha}\left[2 \frac{\partial g_{0 \alpha}}{\partial x^{0}}-\frac{\partial g_{00}}{\partial x^{\alpha}}\right]=\frac{1}{2} \frac{1}{g_{\alpha \alpha}}\left(-\frac{\partial g_{00}}{\partial x^{\alpha}}\right) \\
& =\frac{1}{2\left(\eta_{\alpha \alpha}+h_{\alpha \alpha}\right)}\left(-\frac{\partial h_{00}}{\partial x^{\alpha}}\right)
\end{aligned}
$$

Now,

$\eta_{\alpha \alpha}=-1[$ For $\alpha=1,2,3]$

$\therefore \Gamma_{00}^{\alpha}=\frac{1}{2\left(-1+h_{\alpha \alpha}\right)}\left(-\frac{\partial h_{00}}{\partial x^{\alpha}}\right)$

$=\frac{1}{2}\left(1-h_{\alpha \alpha}\right)^{-1} \frac{\partial h_{00}}{\partial x^{\alpha}}$

Or,

$$
\Gamma_{00}^{\alpha} \approx \frac{1}{2} \frac{\partial h_{00}}{\partial x^{\alpha}} .
$$

UniversePG I www.universepg.com
We have

$$
\frac{d^{2} x^{\alpha}}{d t^{2}}=-\frac{c^{2}}{2} \frac{\partial h_{00}}{\partial x^{\alpha}} ;(\alpha=1,2,3)
$$

Now from Newton's equation of motion we have,

$$
\frac{d^{2} x^{\alpha}}{d t^{2}}+\frac{\partial \Phi}{\partial x^{\alpha}}=0 ;(\alpha=1,2,3)
$$

Where, $\Phi=\frac{G M}{r}$ is the gravitational potential.

Now, acceleration due to gravity is,

$$
\vec{g}=-\nabla \Phi=-\Phi_{, \alpha}=\frac{\partial \Phi}{\partial x^{\alpha}}
$$

From (38) and (39) we have,

$-\frac{c^{2}}{2} \frac{\partial h_{00}}{\partial x^{\alpha}}=-\frac{\partial \Phi}{\partial x^{\alpha}}$

Integrating

$$
\begin{aligned}
& \int \frac{\partial h_{00}}{\partial x^{\alpha}} d x^{\alpha}=\frac{2}{c^{2}} \int \frac{\partial \Phi}{\partial x^{\alpha}} d x^{\alpha} \\
& \Rightarrow 1+h_{00}=\frac{2 \Phi}{\mathrm{c}^{2}}+k(\text { constant }) \Rightarrow g_{00}=\frac{2 \Phi}{\mathrm{c}^{2}}+k
\end{aligned}
$$

Since in the flat space metric component $g=1$ and gravitational potential $\Phi=0$. Hence we have $k=1$.

So, we have,

$$
g_{00}=1+\frac{2 \Phi}{c^{2}} .
$$

This equation implies that Einstein laws of gravitation coincide to the Newton's laws of gravitation.

\section{CONCLUSION:}

In this paper, we try to represent an overview of the metric of space-time curvature in a weak gravitational field and it is a consequence in Newtonian approximation. Confidently we can say, this is valuable and usable and future we may try to more work on it.

\section{ACKNOWLEDGEMENTS:}

First of all, I must thank the Almighty upon who rests the supreme authority. I would like to express my heartfelt gratitude to responsible parents, honorable teachers, and classmates for the guidance, inspiration, and constructive suggestion that helped me in the preparation and accomplishment.

\section{CONFLICT OF INTERESTS:}

The author (s) declared no potential conflicts of the interest with respect to the present research work. 


\section{REFERENCES:}

1. Alam N., and Alam M. (2020). The trend of different parameters for designing integrated circuits from 1973 to 2019 and linked to Moore's law, Aust. J. Eng. Innov. Technol., 2(2), 16-23. https://doi.org/10.34104/ajeit.020.016023

2. Anderson, J.D., Espoito, P.B., Martin, W., and Thornton, C. L. (1975). 'Experimental test of general relativity using time-delay data from Mariner 6 and mariner 7, 'Astrophys. J., 200, 221-32.

3. Ansari, Md. Abdullah, (2020). Tensor: TATTYA AND BABOHAR (Tensor: Theory and Application), Bangla Academy Press, Dhaka, Bangladesh, ISBN 984-07-4085-7.

4. Boss, S.K. (1980). An Introduction to General Relativity. Wiley Eastern Limited. New Delhi: 110002. ISBN 0852260776.

5. Caroll, S. (1997). "Lecture Notes on General Relativity”, gr-qc/9712019. Pp. 1-238. https://arxiv.org/pdf/gr-qc/9712019.pdf

6. Clarke, C. (1929). Elementary General Relativity. Edward Arnold Publishers, London.

7. Ellis, G. F. R. (1988). Williams, R.M. Flat and Curved Space-Times, Oxford University Press, Oxford, England.

https://www.waterstones.com/book/flat-and-curvedspace-times/g-f-r-ellis/r-m-williams/9780198506577

8. Foster, J. and Nightingale, J. D. (1994). A short course in General Relativity, Third Edition Springer, Brighton. ISBN-10:0-387-26078-1.

9. Goyal, J.K and Gupta, K.P. (1942). Theory of Relativity (Special and General), Meerut-250 001 (U.P). India.

https://www.mycoursebook.in/theory-of-relativityjk-goyal-kp-gupta-krishna-prakashan-media-pvt$\underline{\text { Itd.html }}$

10. Hughton, L. P. and K. P. (1990). An Introduction to general Relativity. Cambridge University Press, USA.

https://archive.org/details/introductiontoge0000hugh

11. Max, B. (1964). Einsteins's Theory of Relativity, Dover Publications, ISBN 0486607690.

12. Narlikar, J. V. (1978). General Relativity and Cosmology. Tata Institute of Fundamental Research, Bombay, Balaka.

13. Ohanian, H., and Ruffini, R. (1994). "Gravitation and Space-time”, Norton \& Ompany. https://doi.org/10.1017/CBO9781139003391

14. Resnick, R. (1968). Introduction to Special Relativity, John Wiely \& Sons, Inc Sydney.

15. Richard, L. F. (1983). Differential Geometry and Relativity Theory an Introduction. Marcel Dekker, IN. New York and Basel, ISBN 0-8247-1749-X.

16. Rinder, W. (2001). Relativity, Oxford Univ. Press, Oxford, USA.

https://global.oup.com/academic/product/relativity9780198567325?cc=id\&lang=en\&

17. Stephani, H. (2004). Introduction to Special and General Relativity, Third Edition, Cambridge Univ. Press, ISBN 0521010691.

18. Synge, J.L. and Schild. A. (1949). Tensor Calculus. University of Torneto Press, Toroto, Canada.

19. Veltman, M., (1993). Quantum Theory of Gravitation, in: Methods in Field Theory XXVIII Les Ousches Summer school, eds. R. Balian and J. Zinn-Justin (North-Holland). Methods in Field Theory, pp. 265-328 https://doi.org/10.1142/9789814412674_0007

20. Weinberg, S. (1972). Gravitational and Cosmology: Principles and Applications of General Theory of Relativity, Wiley York.

21. Will, C. M. (2018). Theory and Gravitational Physics, Cambridge Univ. Press, Revised edition. Pp. 350. https://www.twirpx.com/file/1953006/

Citation: Iqbal MA, Mamun AMA, Hossain MR, and Hassan MK. (2020). The metric of space-time curvature in a weak gravitational field and it's consequence in Newtonian approximation, Int. J. Mat. Math. Sci., 2(5), 8792. https://doi.org/10.34104/ijmms.020.087092 @ $\underset{\mathrm{Er}}{\text { @ }}$ 